\title{
A review on link adaptation techniques for energy efficiency and QoS in IEEE802.11 WLAN
}

\author{
Aliya Syahira Mohd Anuar, Wan Norsyafizan W Muhamad, Darmawaty Mohd Ali, \\ Suzi Seroja Sarnin, Norfishah Ab Wahab \\ Faculty of Electrical Engineering, Universiti Teknologi MARA, Malaysia
}

\begin{tabular}{|c|c|}
\hline Article Info & ABSTRACT \\
\hline Article history: & \multirow{13}{*}{$\begin{array}{l}\text { Link adaptation is a technique that able to adapt modulation and coding } \\
\text { scheme (MCS) based on radio channel conditions. With the exponential } \\
\text { increase on the wireless devices nowadays, it contributes to high energy } \\
\text { consumption and an increase in carbon dioxide (CO2) emission which } \\
\text { contribute to the environmental issue. Researchers have developed proposals } \\
\text { to tackle these issues by design algorithms based on link adaptation } \\
\text { technique. Nowadays, various link adaptation techniques have been proposed } \\
\text { by researchers with target for either Quality of Service (QoS) enhancement } \\
\text { as well as energy efficiency. This paper presents A Review on Link } \\
\text { Adaptation Techniques for Energy Efficiency and QoS in IEEE } 802.11 \\
\text { WLAN. In this study, a comprehensive review of the relevant literature } \\
\text { published that focus on link adaptation technique in IEEE } 802.11 \text { WLAN in } \\
\text { improving the energy efficiency and maximize the QoS performance is } \\
\text { presented. Link adaptation can be categorized into transmission power } \\
\text { control adaptation, transmission data rate adaptation and joint rate adaptation. } \\
\text { These adaptations are carried out according to the channel state information } \\
\text { (CSI). CSI can be categorized into signal-to-noise ratio (SNR), bit error rate } \\
\text { (BER), delay, and queue length. }\end{array}$} \\
\hline Received Jan 10, 2019 & \\
\hline Revised May 1, 2019 & \\
\hline Accepted Jul 1, 2019 & \\
\hline Keywords: & \\
\hline Energy saving & \\
\hline IEEE 80211 ax & \\
\hline IEEE 8UL.11AX & \\
\hline Link adaptation & \\
\hline Quality of service (QoS) & \\
\hline WLAN & \\
\hline & \\
\hline & \\
\hline
\end{tabular}

Copyright $@ 2020$ Institute of Advanced Engineering and Science. All rights reserved.

\section{Corresponding Author:}

Aliya Syahira Mohd Anuar, Faculty of Electrical Engineering, Universiti Teknologi MARA(UiTM), 40450 Shah Alam, Selangor Darul Ehsan, Malaysia.

Email: aliyasyahira93@gmail.com

\section{INTRODUCTION}

IEEE 802.11 WLAN is a wireless accessible communication between multiple telecommunication devices by a network connection especially for indoor networking. According to the Cisco in Global Mobile Data Traffic Forecast Update 2011-2016 [1], in year 2019, the number of information and communication technology (ICT) and WLAN will increase due to user's demand and expectation where it increase to the exponential growth of internet traffic [1-3].

Rapid growth of internet traffic could increase energy consumption and carbon footprint which contribute to economy and environmental effects. According to the Inter governmental Panel on Climate Change in Global Emission by Economic Sector that electricity and heat production contribute higher of $\mathrm{CO} 2$ emission as compared to other sectors such as agriculture, forestry, building, and other energy [3]. This is related due to the increment of ICT usage.

In order to resolve the energy consumption issue, there are many techniques proposed which focus on different approaches such as packet size adaptation [4-6], contention window (CW) adaptation [7, 8], passive sleep mode (PSM) [9, 10], and link adaptation [7, 11-15]. 
This paper presents A Review on Link Adaptation Techniques for Energy Efficiency and QoS in IEEE802.11 WLAN. In this study, a comprehensive review of the relevant literature published that focus on link adaptation technique in IEEE 802.11 WLAN in improving the energy efficiency and maximize the QoS performance. Link adaptation can be categorized into transmission power control adaptation, transmission data rate adaptation and joint rate adaptation. The transmission data rate adaptation focus in improving the QoS performances by maximizing the transmission data rate, the transmission power control is one of the approaches for energy efficiency, while the joint rate adaptation consists of the transmission rate adaptation and the transmission power adaptation with aim to improve QoS performance and energy efficiency in a network. These adaptations are carried out according to the channel state information (CSI). CSI is a channel properties in communication link that can be categorized into signal-to-noise ratio (SNR), bit error rate (BER), delay, and queue length.

\section{IEEE 802.11 WLAN}

IEEE 802.11 WLAN is a set of PHY and MAC that widely deployed in telecommunication nowadays. Boris B. [16] stated that in 2020-2030 decade, the number of Information and Communication Technology (ICT), traffic characteristics and user demands encourage the development of WLAN new standard. Figure 1 shows the timeline of released IEEE802.11 WLAN amendments [17]. Based on this figure, it can be seen that amendments of IEEE802.11 WLAN are developed by year to year with the new specification in order to fulfill users' demand.

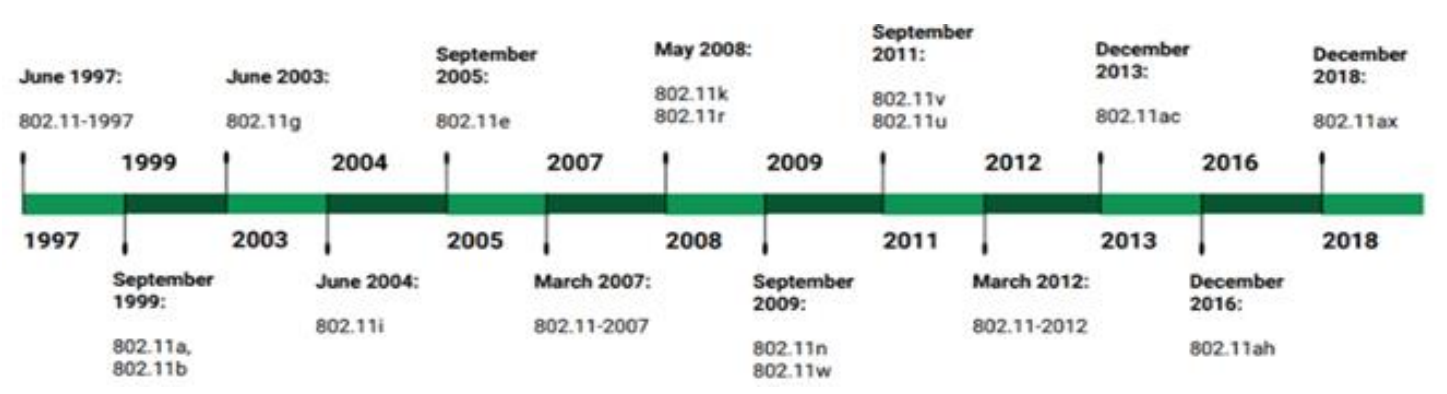

Figure 1. Timeline of IEEE 802.11 WLAN amendments [17]

The first IEEE 802.11 was launched in 1997 and followed by new amendments with some improvement. The successful of IEEE802.11-1997 commence to the development of amendments with specification [18]. IEEE 802.11 amendments is developed by IEEE Standards Association (IEEE SA). IEEE SA responsible to assemble a broad stakeholder community [19]. The most famous amendment in IEEE 802.11 WLAN is IEEE $802.11 \mathrm{a} / \mathrm{b} / \mathrm{g}$ due to its application in WLAN and able to provides $11 \mathrm{Mbps}$ transmission [17]. The invention of IEEE $802.11 \mathrm{a} / \mathrm{b}$ have enhanced the development of new amendments with improvement in specification. For instead, IEEE $802.11 \mathrm{~g}$ with 54Mbps was launched in 2003. The advantage of IEEE 802.11g are higher power and better coverage [20]. As time flies, the new fifth generation of IEEE 802.11 WLAN, IEEE 802.11ac was created with very high throughput up to $1 \mathrm{~GB}$.

IEEE 802.11ac also describe as Very High Throughput $6 \mathrm{GHz}$ was released in December 2013. It is known so because of the specification in providing high throughput of WLAN on $5 \mathrm{GHz}$ band. As compared to the previous amendments, such as $802.11 \mathrm{a} / \mathrm{b} / \mathrm{g}$, and $802.11 \mathrm{n}$, it shows that IEEE 802.11 ac have higher modulation scheme which up to 256 quadrature amplitude modulation (QAM) where it is a key role in the size of throughput. The performance of IEEE 802.11ac have a higher throughput compare to IEEE 802.11n WLAN [13, 21]. Following the IEEE 802.11ac, is IEEE 802.11ax. IEEE802.11ax also known as WiFi 6 is a successor to current IEEE 802.11ac amendments with high efficiency WLAN. Details of link adaptation technique will be discussed in the following section.

\section{LINK ADAPTATION}

Adaptive modulation and coding scheme (AMCS) which also known as link adaptation is a technique where it able to adapt modulation scheme and coding rate (MCS) based on the conditions of radio channel [13]. Figure 2 illustrates the link adaptation method by adapting modulation scheme and coding rate of error correction in radio link. Based on this figure, a good radio channel with high throughput is affected 
by a high modulation scheme and smidgen of error detection. If the number of modulation scheme low and large amount of error detection, it affecting radio channel to low data throughput.

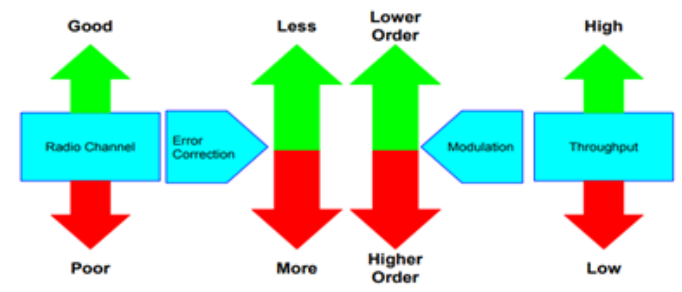

Figure 2. Method of link adaptation

Method of link adaptation is used due to its ability in improving network performance [22]. In good channel condition, the modulation scheme is high-level, and small quantity of error correction is used, where it contributes to high-data throughput. Meanwhile, during congested traffic channel, there will be large quantity of error correction, and low-level of modulation scheme where it contributes to low data throughput.

As mentioned, the link adaptation approach consists of the transmission rate adaptation as well as the transmission power control (TPC) and the transmission joint rate adaptation as shown in Figure 3 . The TPC is a mechanism in IEEE 802.11 WLAN used to reduce the transmit power and prolong the battery of the device [23]. Radio channel condition can be estimated based on channel state information (CSI). Figure 3 shows the classification of link adaptation techniques with variety of CSI that the technique can be used. CSI is channel properties in communication link that can be categorized to signal-to-noise ratio (SNR), bit error rate (BER), delay, and queue length as shown in this figure.

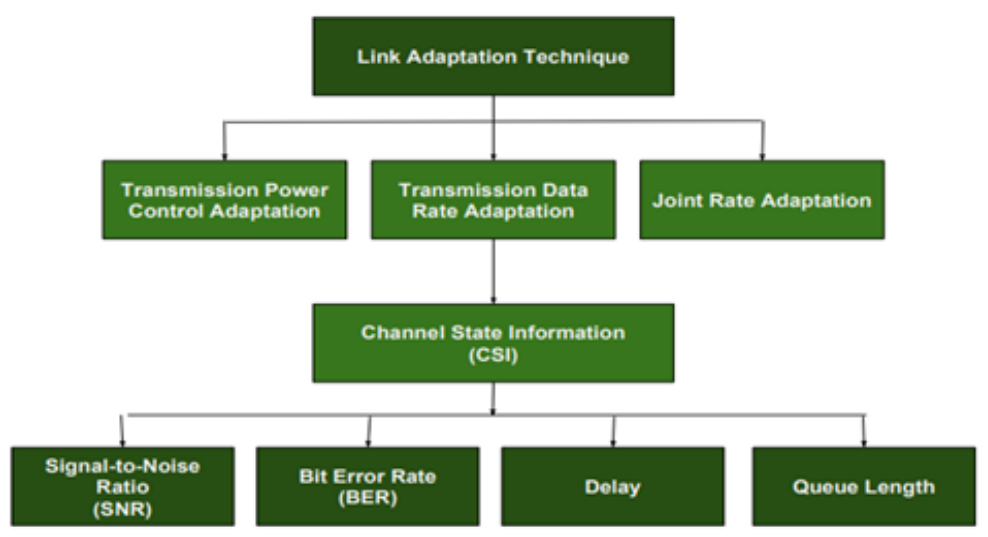

Figure 3. Classification of link adaptation technique

\subsection{Transmission Rate Adaptation}

According to Zhang in his research work [24], transmission rate adaptation is a link layer mechanism that represent the performance of communication link especially in IEEE 802.11 WLAN. The performance of transmission data rate is affected by modulation and coding scheme (MCS).

Radio wave is ease affected by interference such as fading and pathloss. The ability of MCS in adapting the condition of radio link makes it crucial in determine the performance of radio link. The higher modulation scheme, the lower amount of error correction, the higher throughput can be achieved by receiver, where it provides a good radio channel. If the number of modulation scheme low and amount of error detection is large, it will affect the performance of radio channel to low data throughput.

Table 1 represent the number of data rate in each modulation scheme for one spatial stream (SS) in IEEE 802.11ax WLAN. Based on this table, it can be seen that the highest modulation scheme, the highest number of data rate, which is it provide a good radio link. It also shows that the next generation of IEEE 802.11ax WLAN will have $1020.80 \mathrm{Mbps}$ data rate at $160 \mathrm{MHz}$ with one SS, higher than IEEE 802.11ac.

Table 1. IEEE 802.11ax WLAN Modulation and coding schemes (MCS) for single spatial stream

A review on link adaptation techniques for energy efficiency and QoS in ... (Aliya Syahira Mohd Anuar) 


\begin{tabular}{lllllll}
\hline & & \multicolumn{5}{c}{ Data Rate in Mbps } \\
MCS & Modulation & Coding Rate & $20 \mathrm{MHz}$ & $40 \mathrm{MHz}$ & $80 \mathrm{MHz}$ & $160 \mathrm{MHz}$ \\
\hline 0 & BPSK & $1 / 4$ & 3.60 & 7.30 & 15.30 & 30.60 \\
0 & BPSK & $1 / 2$ & 7.30 & 14.60 & 30.60 & 61.30 \\
1 & QPSK & $1 / 4$ & 7.30 & 14.60 & 30.60 & 61.30 \\
1 & QPSK & $1 / 2$ & 14.60 & 29.30 & 61.30 & 122.50 \\
2 & QPSK & $3 / 4$ & 21.90 & 43.90 & 91.90 & 183.80 \\
3 & 16-QAM & $1 / 4$ & 14.60 & 29.30 & 61.30 & 122.50 \\
3 & 16-QAM & $1 / 2$ & 29.30 & 58.50 & 122.50 & 245.00 \\
4 & 16-QAM & $3 / 8$ & 21.90 & 43.90 & 91.90 & 183.80 \\
4 & 16-QAM & $3 / 4$ & 43.90 & 87.80 & 183.80 & 367.50 \\
5 & 64-QAM & $2 / 3$ & 58.50 & 117.00 & 245.00 & 490.00 \\
6 & 64-QAM & $3 / 4$ & 65.80 & 131.60 & 275.60 & 551.50 \\
7 & 64-QAM & $5 / 6$ & 73.10 & 146.30 & 306.30 & 612.50 \\
8 & 256-QAM & $3 / 4$ & 87.80 & 175.50 & 367.50 & 735.00 \\
9 & 256-QAM & $5 / 6$ & 97.50 & 195.00 & 408.30 & 816.60 \\
10 & 1024-QAM & $3 / 4$ & 109.70 & 219.40 & 459.40 & 918.80 \\
11 & 1024-QAM & $5 / 6$ & 121.90 & 243.80 & 510.40 & 1020.80 \\
\hline
\end{tabular}

\subsection{Transmission Power Control Adaptation}

Transmission Power Control (TPC) is a mechanism that used in IEEE 802.11 WLAN to improve the power management by allowing a WLAN device to use the minimum required power level during transmission and is complimentary to the power management policies. In order to determine the appropriate transmit power, a wireless station needs to estimate the link quality between itself and the receiver [25].

\subsection{Joint Rate Adaptation}

Joint Rate Adaptation is a combination between transmission data rate adaptation and transmission power control adaptation in link adaptation. Most of the existing works proposed transmission data rate adaptation and power control adaptation individually as both adaptations are focused on two different goals which are either improving throughput or saving energy consumption. In order to maximize the throughput, it is necessary to operate in higher level of modulation with fewer redundant bits in coding, thus requires higher transmission power to maintain acceptable bit error rate (BER) performance. Therefore, how to achieve performance trade-off between energy efficiency and QoS performance becomes a difficult challenge in the design of joint rate and power adaptation.

\section{EXISTING TECHNIQUES}

Adaptation of transmission data rate is one of the link adaptation techniques with purpose to dynamically increase the data rate. Table 2 is a summary of previous researches that deals with the link adaptation technique either transmission rate adaptation, transmission power control adaptation or joint rate adaptation in their researches. Many researchers propose approaches of adaptive modulation and coding scheme in their researches [7, 11, 24, 26-28, 10, 29]. For instance, Raja K. et al proposed a closed-loop, cross-layer and link adaptation algorithm in Dynamic Link Adaptation for High Throughput Wireless Access Networks [11], where the model named as HiWiLA (High Throughput Wireless Link Adaptation) were used to increase throughput in wireless network by using received signal strength indicator (RSSI) and MC throughput as the adaptation parameters. The authors proved that HiWiLA model increase the throughput better than existing schemes such as Minstrel, Minstrel HT, and SampleLite by average $10 \%$ to $70 \%$ of throughput enhancement.

In IEEE 802.11ac Link Adaptation Under Mobility presented by Raja K. et al [30], the authors proposed HT-MobiRate (High Throughput Mobility Rate) a closed-loop mechanism which exploited using Thompson Sampling in dynamic link adaptation under mobile scenario. In this research, authors proposed multi-armed bandit framework because in mobile environment, the channel condition is fluctuating either good channel and bad channel. Due to this issue, authors apply Thompson Sampling technique in this multiarmed bandit framework for HT-MobiRate. The rate of exploration and exploitation in this model dynamically updates with respect to the probability distribution of each link configuration. The results proved that HT-MobiRate model performs better than other schemes especially for link adaptation in static environment.

Dynamic RLC Mode Base Upon Link Adaptation to Reduce Latency and Improve Throughput in Cellular Network [48] proposed by Ritesh S. and Ricardo S. C. is one of amongst researches that used transmission rate adaptation. In this paper, authors proposed modalities of Radio Link Control (RLC) protocol which are Unknowledgement Mode (UM) and Acknowledgement Mode (AM). These two modes 
are suitable for data traffic in improving the system latency as well as the throughput. Based on the comparison between UM and AM that authors mentioned in this paper, it is better to use AM rather than UM in transferring large file. UM function is to detect the packet loss and its performance is depending on the transmission control protocol (TCP) congestion control instantaneous state upon occurrence of the packet loss [31]. By proposing dynamic selection of RLC protocol; UM and AM, which adapts to the radio channel condition, the approached able to minimize the system latency as well as maintaining the throughput in the LTE network.

Transmission power control (TPC) adaptation also one of the link adaptation techniques to improve energy efficiency where it minimizes the transmit power to transmit at low data rate. In literature, there are many works focus on TPC as presented by various works [32, 9, 22, 28, 33-37]. With focus to optimize the power consumption without affecting the network performance parameters such as delay, throughput and jitter, paper "Adaptive Modulation for Energy Efficiency in Wireless Communication” by Yashaswini C. S. et al proposed transmission power adaptation approached adapts to the BER in different modulation techniques which are differential encoded binary phase-shift keying (DBPSK), quadrature phase-shift keying (QPSK), and quadrature amplitude modulation (QAM) [36]. In this paper, the proposed system will select the best modulation either DBPSK, QPSK and QAM, when the amount of BER increase and SNR decrease which is in bad channel condition. On the other hand, in good channel condition where the BER in decrease and SNR increase, the modulation is remained. This adaptive modulation technique has achieved the objective of this research which are optimize energy consumption as well as maintain the QOS in the network.

Power consumption issue also attract researcher to apply in medical application, such as in paper "Energy Efficiency Comparison Between Data Rate Control and Transmission Power Control Algorithms for Wireless Body Sensor Networks" by Ali H. S. where the authors compared the result of energy efficiency between the transmission data rate control and the transmission power control [37]. Based on this paper, there are 2 cases study have been presented which are i) increase data rate and keep transmission power low in high peak variable data rates, and ii) increase transmission power level and keep data rates constant in high transmission power consumption. RSSI is used as the adapt parameter in developing the algorithms; video transmission rate control algorithm (VTRCA) and transmission power control algorithm. In developing VTRCA, the RSSI value is stable to ensure the transmission power in high peak variable data rates case is constant, while in developing transmission power control algorithm, the RSSI value is deteriorate in high transmission power consumption case to maximize the energy efficiency. The authors conclude that VTRCA is saving more energy about $47.8 \%$ compared to the transmission power control algorithm with energy saving of $40.9 \%$ in the WBSNs.

Joint Rate Adaptation is a combination between transmission data rate adaptation and transmission power control adaptation in link adaptation. Most of the researches are focus only either transmission data rate or transmission power control. However, there are few works proposed joint adaptation in their researches [7], [28, 38, 39]. Research paper by Saeed A. and Steven D. B. in Joint Rate Adaptation, Frame Aggregation and MIMO Mode Selection for IEEE 802.11ac [38] proposed a joint rate adaptation by joining the data rate, MIMO mode and frame aggregation configuration at the access point (AP) with target to maximize the throughput performance in IEEE 802.11ac WLAN. This research focused at MAC layer because there are frame aggregation capabilities in MAC layer which are Aggregated-MAC Service Data Unit (A-MSDU) and Aggregated-MAC Protocol Data Unit (A-MPDU), that able to reduce the transmission overhead as well as improve the MAC layer efficiency. By using the first-event error probability of bit error rate (BER) as adaptation parameter in joint selection scheme, objective of the proposed technique achieved significant improvements in throughput of IEEE 802.11ac WLAN.

Another work that discussed the joint rate adaptation which are transmission power adaptation, channel adaptation and data rate adaptation, is "The Impact of Link Adaptation on WiFi 802.11n" which evaluate the IEEE 802.11n network throughput's performance, delay, and medium access delay [39]. In this paper, transmission power adaptation is applied for save energy consumption. However, in the case where received signal too weak, the transmit power need to be increased to ensure the packet or information data is successfully received at the receiver. Channel adaptation approached in this paper is when the channel condition is congested, the packets will switch to another channel which less congested channel to ensure the packet received at the receiver. Meanwhile, rate adaption is applied when the channel condition is good and the SNR is high, the AP will switch to another MCS which available in the IEEE 802.11n that offers a higher data rate, and in the same time can reduce the energy consumption [39]. The authors shows that link adaptation algorithm approached can greatly improve WiFi network performance increased in throughput and WLAN delay drops by $60 \%$ [39]. Although this paper used transmission power adaptation approached, the authors did not focus much on the power consumption in the network as much as delay and throughput because their main objective is to improve the network performance. 
Based on existing research works as discussed, it can be concluded that, most of the proposed link adaptation techniques targeted to improve the throughput and delay without considering much on power consumption. Based on the finding, in future the new link adaptation technique will be designed to improve the energy efficiency and maintaining the QoS via IEEE 802.11ax WLAN.

Table 2. Summary of related research studies

\begin{tabular}{|c|c|c|c|c|c|c|c|}
\hline No & Author & Paper (Year) & Techniques & Network & $\begin{array}{l}\text { Performance } \\
\text { Metric }\end{array}$ & CSI & Results \\
\hline 1 & $\begin{array}{l}\text { Saeed A., } \\
\text { Steven D. } \\
\text { B.[38] }\end{array}$ & $\begin{array}{l}\text { Joint Rate Adaptation, } \\
\text { Frame Aggregation } \\
\text { and MIMO Selection } \\
\text { for IEEE 802.11ac } \\
(2016)\end{array}$ & $\begin{array}{l}\text { Jointly selects } \\
\text { MCS, the } \\
\text { MIMO mode } \\
\text { and aggregation }\end{array}$ & $\begin{array}{l}\text { IEEE } \\
802.11 \mathrm{ac}\end{array}$ & Throughput & BER & $\begin{array}{l}\text { Significant } \\
\text { improvements } \\
\text { in throughput }\end{array}$ \\
\hline 2 & $\begin{array}{l}\text { Raja K., } \\
\text { Samiran } \\
\text { C., } \\
\text { Sandip } \\
\text { C.[11] }\end{array}$ & $\begin{array}{lr}\text { Dynamic } & \text { Link } \\
\text { Adaptation } & \text { for High } \\
\text { Throughput } & \text { Wireless } \\
\text { Access } & \text { Networks } \\
(2015) & \end{array}$ & \begin{tabular}{lr}
\multicolumn{2}{l}{ Transmission } \\
Rate Closed \\
loop link \\
adaptation
\end{tabular} & $\begin{array}{l}\text { IEEE } \\
80211 \mathrm{n} \text { and } \\
\text { IEEE802.1 } \\
1 \mathrm{ac}\end{array}$ & Throughput & $\begin{array}{l}\text { RSSI and } \\
\text { MAC } \\
\text { throughput }\end{array}$ & $\begin{array}{l}\text { Achieve on } \\
\text { average } 10 \%- \\
70 \% \text { better } \\
\text { throughput than } \\
\text { existing state of } \\
\text { the art schemes }\end{array}$ \\
\hline 3 & $\begin{array}{l}\text { Raja K., } \\
\text { Samiran } \\
\text { C., } \\
\text { Sandip } \\
\text { C.[30] }\end{array}$ & $\begin{array}{lr}\text { IEEE } 802.11 \mathrm{ac} & \text { Link } \\
\text { Adaptation } & \text { under } \\
\text { Mobility (2017) } & \end{array}$ & $\begin{array}{l}\text { Transmission } \\
\text { Rate adaptation }\end{array}$ & $\begin{array}{l}\text { IEEE } \\
802.11 \mathrm{ac}\end{array}$ & Throughput & SNR & $\begin{array}{ll}\text { Enhance } & \\
\text { network } & \\
\text { performance } & \text { in } \\
\text { terms } & \text { of } \\
\text { throughput } \\
\text { compared to } \\
\text { other } \\
\text { techniques. } \\
\text { Minimizes }\end{array}$ \\
\hline 4 & $\begin{array}{l}\text { Ritesh S., } \\
\text { Ricardo } \\
\text { S. C.[35] }\end{array}$ & $\begin{array}{l}\text { Dynamic RLC Mode } \\
\text { Based Upon Link } \\
\text { Adaptation to Reduce } \\
\text { Latency and Improve } \\
\text { Throughput in Cellular } \\
\text { Networks (2016) }\end{array}$ & $\begin{array}{l}\text { Transmission } \\
\text { Rate adaptation }\end{array}$ & LTE & $\begin{array}{l}\text { Delay and } \\
\text { throughput }\end{array}$ & $\begin{array}{l}\text { BER and } \\
\text { signal-to- } \\
\text { interference } \\
\text { ratio (SIR) }\end{array}$ & $\begin{array}{l}\text { system delay by } \\
\text { reducing } \\
\text { overhead and } \\
\text { complexity } \\
\text { whilst } \\
\text { maintaining } \\
\text { data reliability. }\end{array}$ \\
\hline 5 & $\begin{array}{l}\text { Yashaswi } \\
\text { ni C. S. } \\
\text { et al }[36]\end{array}$ & $\begin{array}{l}\text { Adaptive Modulation } \\
\text { for Energy Efficiency } \\
\text { in Wireless } \\
\text { Communication } \\
(2018)\end{array}$ & TPC & $\begin{array}{l}\text { General } \\
\text { Wireless } \\
\text { Network }\end{array}$ & $\begin{array}{l}\text { Power and } \\
\text { throughput }\end{array}$ & $\begin{array}{l}\text { BER and } \\
\text { SNR }\end{array}$ & $\begin{array}{l}\text { The power } \\
\text { consumption is } \\
\text { optimized and } \\
\text { number of } \\
\text { retransmissions } \\
\text { in the network } \\
\text { is decreased. }\end{array}$ \\
\hline 7 & $\begin{array}{l}\text { Ali H. S. } \\
\text { et al [37] }\end{array}$ & $\begin{array}{l}\text { Energy Efficiency } \\
\text { Comparison Between } \\
\text { Data Rate Control and } \\
\text { Transmission Power } \\
\text { Control Algorithms for } \\
\text { Wireless Body Sensor } \\
\text { Networks (2018) }\end{array}$ & $\begin{array}{l}\text { Joint Rate } \\
\text { Adaptation }\end{array}$ & $\begin{array}{l}\text { Wireless } \\
\text { Body } \\
\text { Sensor } \\
\text { Networks }\end{array}$ & $\begin{array}{l}\text { Throughput and } \\
\text { power }\end{array}$ & RSSI & $\begin{array}{l}\text { VTRCA save } \\
\text { more power } \\
\text { about } 47.8 \% \\
\text { compared to the } \\
\text { transmission } \\
\text { power control } \\
\text { algorithm with } \\
\text { energy saving } \\
\text { of } 40.9 \% \text { in the } \\
\text { WBSNs. }\end{array}$ \\
\hline
\end{tabular}

\section{CONCLUSION}

This paper reviewed published literature with the target to enhance QoS as well as energy consumption specifically via link adaptation technique in IEEE 802.11 WLAN. The ability of link adaptation in adapting the MCS based on radio channel conditions make it desirable to enhance QoS and energy efficiency in a network. Based on the review, link adaptation that has been studied by previous researches are aimed on the throughput and delay without considering energy consumption. Due to this research gap, the link adaptation algorithm with focus to enhance energy efficiency and QoS in IEEE 802.11ax WLAN will be selected for future work due to its specifications which are high data rate and energy efficiency. Thus, this technique will help in reducing operating cost and improving society health by reducing the greenhouse effect, carbon footprint and depletion of the ozone layer. 


\section{ACKNOWLEDGEMENTS}

This research was supported under budget of Grant GIP, University Teknologi MARA, Malaysia.

\section{REFERENCES}

[1] Cisco, "Cisco Visual Networking Index: Global Mobile Data Traffic Forecast Update, 2011-2016\&nbsp; [Visual Networking Index (VNI)]," Cisco, pp. 2016-2021, 2012.

[2] Agency for Natural Resources and Energy, "Key World Energy statistics," IEA Int. Energy Agency, 2017.

[3] Intergovernmental Panel on Climate Change., O. Edenhofer, and Intergovernmental Panel on Climate Change. Working Group III, Climate change 2014 : mitigation of climate change : Working Group III contribution to the Fifth assessment report of the Intergovernmental Panel on Climate Change.

[4] Y. Nomura, K. Mori, and H. Kobayashi, "Efficient Frame Aggregation with Frame Size Adaptation for Next Generation MU-MIMO WLANs," 2015.

[5] M. Kim, C. Choi, A. Ru, K. Vshhg, and Q. Z. H. Sursrvh, "Joint Rate and Fragment Size Adaptation in IEEE 802. 11n Wireless LANs," pp. 942-947, 2011.

[6] D. Library et al., "Packet size based performance analysis of IEEE 802. 11 WLAN comprising virtual server arrays," pp. 1-3, 2018.

[7] T. Y. Lin, C. Y. Tsai, and K. R. Wu, "EARC: Enhanced adaptation of link rate and contention window for IEEE 802.11 multi-rate wireless networks," IEEE Trans. Commun., vol. 60, no. 9, pp. 2623-2634, 2012.

[8] Q. Dong, K. Hayashi, and M. Kaneko, "A New Adaptive Modulation and Coding Method for CommunicationBased Train Control Systems using WLAN," IFAC-PapersOnLine, vol. 49, no. 22, pp. 139-144, 2016.

[9] D. P. Consumption et al., "Demystifying 802.11n Power Consumption," Proc. 2010 Work. Power Aware Comput. Syst., p. 1, 2010.

[10] W. N. W. Muhamad, J. Y. Khan, and J. Brown, "A QoS guaranteed low energy packet transmission technique for the IEEE 802.11 WLAN,” 2014 9th Int. Symp. Commun. Syst. Networks Digit. Signal Process. CSNDSP 2014, pp. 617-622, 2014.

[11] R. Karmakar, S. Chattopadhyay, and S. Chakraborty, "Dynamic Link Adaptation for High Throughput Wireless Access Networks," pp. 1-6, 2015.

[12] Y. Sun et al., "Goodput performance of ultrahigh-speed WLAN via link adaptation algorithm," Int. Conf. Commun. Technol. Proceedings, ICCT, pp. 1344-1348, 2010.

[13] L. Caponi, F. Chiti, and R. Fantacci, "Performance Evaluation of a Link Adaptation Technique for High Speed Wireless Communication Systems," IEEE Trans. Wirel. Commun., vol. 6, no. 12, pp. 4568-4575, 2007.

[14] W. Lin et al., "Integrated link-system level simulation platform for the next generation WLAN - IEEE 802.11ax," 2016 IEEE Glob. Commun. Conf. GLOBECOM 2016 - Proc., 2016.

[15] T. Desai and H. Shah, "Energy efficient link adaptation using machine learning techniques for wireless OFDM," 2016 Int. Conf. Inven. Comput. Technol., vol. 3, pp. 1-4, 2016.

[16] B. Bellalta, "IEEE 802.11ax: High-efficiency WLANS," IEEE Wirel. Commun., vol. 23, no. 1, pp. 38-46, 2016.

[17] D. J. Deng, S. Y. Lien, J. Lee, and K. C. Chen, "On Quality-of-Service Provisioning in IEEE 802.11ax WLANs," IEEE Access, vol. 4, pp. 6086-6104, 2016.

[18] M. S. Gast, 802.11ac A Survival Guide. 2013.

[19] Bruce Kraemer, "Creation of new IEEE 802.11TM High Efficiency WLAN Study Group," doc. : IEEE 802.11$13 / 0418 \mathrm{r} 2$.

[20] K. J. Negus and A. Petrick, "History of wireless local area networks (WLANs) in the unlicensed bands," Info, vol. 11 , no. 5, pp. 36-56, 2009.

[21] S. Narayan, C. Jayawardena, J. Wang, W. Ma, and G. Geetu, "Performance test of IEEE 802.11ac wireless devices," 2015 Int. Conf. Comput. Commun. Informatics, ICCCI 2015, pp. 3-8, 2015.

[22] K. Xiao and Y. Zhangt, "eCOPE : Energy Efficient Network Coding Scheme in Multi-rate Wireless Networl (s," no. 2012, pp. 18-23, 2013.

[23] R. Cisco and S. Community, "Prerequisites Requirements."

[24] K. Zhang, A. Lim, S. Wu, and Q. Yang, "A High TCP Performance Rate Adaptation Algorithm for IEEE 802.11 Networks,” Int. J. Comput. Networks Commun., vol. 2, no. 6, pp. 31-44, 2010.

[25] W. N. W. Muhamad, "Enhanced Energy Efficiency Techniques for Packet Transmission in IEEE 802 . 11 Networks," Univ. Newcastle, no. March, 2016.

[26] L. Lopacinski, M. Brzozowski, R. Kraemer, S. Büchner, and J. Nolte, "Improving energy efficiency using a link adaptation algorithm dedicated for 100 Gbps wireless communication,” AEU - Int. J. Electron. Commun., vol. 81, pp. 67-73, 2017.

[27] S. R. Chaudhary, A. J. Patil, and A. V. Yadao, "WLAN-IEEE 802.11ac: Simulation and performance evaluation with MIMO-OFDM,” Conf. Adv. Signal Process. CASP 2016, pp. 440-445, 2016.

[28] O. Narmanlioglu, R. C. Kizilirmak, T. Baykas, and M. Uysal, "Link Adaptation for MIMO OFDM Visible Light Communication Systems," IEEE Access, vol. 5, 2017.

[29] M. Yazid, A. Ksentini, L. Bouallouche-Medjkoune, and D. Aïssani, "Performance analysis of the TXOP sharing mechanism in the VHT IEEE 802.11ac WLANs," IEEE Commun. Lett., vol. 18, no. 9, pp. 1599-1602, 2014.

[30] R. Karmakar, S. Chattopadhyay, and S. Chakraborty, "IEEE 802.11ac Link Adaptation Under Mobility," 2017 IEEE 42nd Conf. Local Comput. Networks, pp. 392-400, 2017.

[31] A. Larmo, M. Lindström, M. Meyer, G. Pelletier, and J. Torsner, "LTE_WP_0904_Ericsson,” no. April, pp. 52-59, 2009. 
[32] D. Feng, C. Jiang, G. Lim, L. J. Cimini, G. Feng, and G. Y. Li, "A survey of energy-efficient wireless communications," IEEE Commun. Surv. Tutorials, vol. 15, no. 1, pp. 167-178, 2013.

[33] M. Imoto, "Global standardization activities of DeviceNet," Proc. 41st SICE Annu. Conf. SICE 2002., vol. 2, pp. 913-916.

[34] R. K. Sheshadri and D. Koutsonikolas, “On Packet Loss Rates in Modern 802. 11 Networks,” Infocom, 2017.

[35] R. Shreevastav and R. S. Carbajo, "Dynamic RLC mode based upon link adaptation to reduce latency and improve throughput in cellular networks," 2016 IEEE 7th Annu. Ubiquitous Comput. Electron. Mob. Commun. Conf. UEMCON 2016, pp. 2-7, 2016.

[36] R. Achary, “Adaptive Modulation for Energy Efficiency in Wireless Communication,” vol. 3, no. 5, pp. 2152$2155,2014$.

[37] A. H. Sodhro, L. Chen, A. Sekhari, Y. Ouzrout, and W. Wu, "Energy efficiency comparison between data rate control and transmission power control algorithms for wireless body sensor networks," Int. J. Distrib. Sens. Networks, vol. 14, no. 1, 2018.

[38] S. Abdallah and S. D. Blostein, "Joint rate adaptation, frame aggregation and MIMO mode selection for IEEE 802.11ac," IEEE Wirel. Commun. Netw. Conf. WCNC, vol. 2016-Septe, no. Wcnc, pp. 1-6, 2016.

[39] H. J. Zhu and D. Kidston, "The impact of link adaptation on Wifi 802.11N," 2016 IEEE Int. Conf. Netw. Archit. Storage, NAS 2016-Proc., 2016.

\section{BIOGRAPHIES OF AUTHORS}
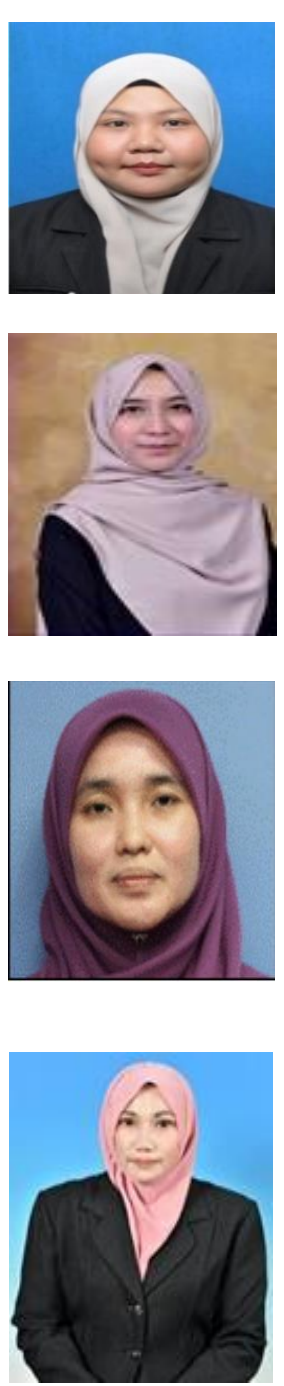

Aliya Syahira Mohd Anuar received her Bachelor of Electronic Engineering from Universiti Teknologi MARA (UiTM), Shah Alam, Malaysia, in July 2017. She is currently a Master student with Universiti Teknologi MARA, Shah Alam, Malaysia. Her research interests include wireless networking and performance optimization.

Wan Norsyafizan W. Muhamad received her Bachelor in Electrical Engineering from the Universiti Malaya, Malaysia in 2002. She completed her Master of Electrical Engineering from Universiti Malaya, Malaysia in 2009. She obtained her Ph.D in 2017 from University of Newcastle, Australia. Currently, she is a Senior Lecturer in Faculty of Electrical Engineering at Universiti Teknologi MARA, Shah Alam, Malaysia. Her current research interest is in the area of wireless communication (Physical and MAC cross layer optimization).

Darmawaty Mohd Ali is an Associate Professor in the Department of Communication Engineering, Universiti Teknologi MARA (UiTM). She obtained her Ph.D in 2012 from Universiti Malaya, Malaysia. She has received the Master of Engineering in Electrical in 2002 from Universiti Teknologi Malaysia. Previously, she obtained her first degree from Universiti Kebangsaan Malaysia with Honours, in Electrical, Electronic and System, graduating in 1999. She is a member of Wireless Communication Technology (WiCOT) Research Interest Group (RIG) and her research interests include Wireless Access Technology and Quality of Service in Wireless Broadband.

Suzi Seroja Sarnin received her Bachelor of Electrical and Electronics (B.Eng), in field of Communication from the Universiti Teknologi Malaysia, Skudai, in 1999. She completed her Master of Microelectronics (Msc), from the Universiti Kebangsaan Malaysia in 2005. She has a $\mathrm{PhD}$ of Electrical Engineering from Universiti Teknologi MARA, Shah Alam, Malaysia, in 2019. As a senior lecturer at Universiti Teknologi MARA she has collaborated actively with another researcher in several other disciplines of Electrical Engineering. Her current research interests are in the area of wireless communication and Internet of Things. 


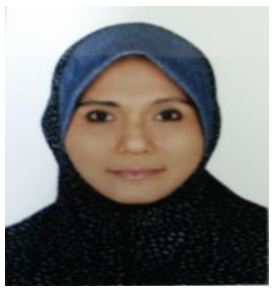

Norfishah Ab Wahab was born in Malaysia, in 1963. She received the degree in Electronics Engineering from Universiti Teknologi MARA (UiTM), Malaysia, MSc in Telecommunication and Information Engineering and $\mathrm{PhD}$ in microwaves, electromagnetism in the same university. Currently, she is a senior lecturer in Faculty of Electrical Engineering, UiTM Shah Alam, Malaysia. She has been working in UiTM since April 2008. From 1992 to 2004, she was employed by Telekom Malaysia. She worked in various divisions such as microwave, fiber cable and network system departments. Her major research areas are in microwave and electromagnetic modeling, low-powered electronic design, energy harvesting and efficiency. 